\title{
An unusual cause of ankle swelling
}

Authors

Institutions
Rhys O. Butcher ${ }^{1}$, Laura Kinsey ${ }^{1}$, Hui Y. Lee ${ }^{1}$, Lucy Foster ${ }^{2}$, Stephen M. McGrath² ${ }^{2}$ Robert P. Willert ${ }^{1}$

${ }^{1}$ Department of Gastroenterology, Central Manchester University Hospitals, Manchester Royal Infirmary, Manchester, United Kingdom

${ }^{2}$ Department of Histopathology, Central Manchester University Hospitals, Manchester Royal Infirmary, Manchester, United Kingdom submitted:

12. May 2014

accepted after revision:

3. June 2014

\section{Bibliography}

DOI http://dx.doi.org/

10.1055/s-0034-1377382

Published online: 1.8.2014

Endoscopy International Open

2014; 02: E262-E264

(c) Georg Thieme Verlag KG

Stuttgart · New York

E-ISSN 2196-9736

\section{Corresponding author}

Robert P. Willert

Department of

Gastroenterology

Central Manchester University

Hospitals

Manchester Royal Infirmary

Oxford Road

Manchester M13 9WL

United Kingdom

Phone: +44-161-2767992

robet.willert@cmft.nhs.uk
License terms

()(1) $\Theta \circledast$

\section{Introduction}

$\nabla$

Primary Intestinal Lymphangiectasia (PIL) is a rare idiopathic congenital disorder characterized by impaired lymphatic drainage and protein-losing enteropathy $[1,2]$.

PIL normally presents in childhood but can unusually present in adults [2]. We report a case of PIL diagnosed at antegrade double-balloon enteroscopy in a middle-aged man with isolated intermittent ankle edema and hypoalbuminemia.

\section{Case report}

$\nabla$

A 51-year-old Caucasian man presented to the Outpatient Gastroenterology clinic with a 1-year history of intermittent ankle swelling. He was otherwise well and denied any gastrointestinal symptoms or weight loss. He had no co-morbidities and regularly ran triathlons. He was a nonsmoker and had no family history of note. Cardiology review had excluded a cardiorespiratory cause for his symptoms.

Physical examination was unremarkable. Laboratory studies revealed an albumin level of $29 \mathrm{~g} / \mathrm{L}$, protein of $58 \mathrm{~g} / \mathrm{L}$, hypogammaglobulinemia (IgG $4.57 \mathrm{~g} / \mathrm{L}$ ) and lymphopenia (lymphocytes $0.98 \times$ $10^{9} / \mathrm{L}$ ). No paraprotein was noted on electrophoresis and remaining bloods including full blood count, renal function, liver function, inflammatory markers, and hematinics were all normal. Celiac serology, autoimmune, and human immunodeficiency virus (HIV) screening were all negative and serum angiotensin-converting enzyme (ACE) was normal.

Urinalysis and urine protein-creatinine ratio were normal.

Gastroscopy was normal but a barium followthrough and computed tomography (CT) of the abdomen revealed possible thickening without mass lesion in the proximal small bowel. Magnet- ic resonance imaging (MRI) of the small bowel confirmed an abnormal mucosal pattern predominantly affecting the jejunum with prominent mucosal folds that showed regular thickening. The patient therefore proceeded to antegrade double-balloon enteroscopy and a thickened jejunal mucosa was seen with visibly enlarged whitetipped villi and free intraluminal lymph that extruded from the mucosa at biopsy ( $\bullet$ Fig. 1,2).

His case was subsequently reviewed at the Gastroenterology Multidisciplinary team (MDT) meeting. Jejunal biopsies revealed dilated mucosal lymphatics with no evidence of Whipple's disease, amyloidosis or granulomas ( Fig. 3). The original CT imaging was also reviewed and the thoracic duct was normal with no secondary obstructive cause seen. The clinical, radiological, endoscopic, and histological findings were felt to be consistent with a diagnosis of Primary Intestinal Lymphangiectasia (PIL).

He was subsequently managed with a low fat, high-protein diet supplemented by mediumchain triglycerides and responded well to these dietary changes with resolution of his hypoalbuminemia and ankle edema. He remained stable over 2 years of follow-up and was able to continue competing in triathlons.

\section{Discussion}

$\nabla$

This case represents an unusual presentation of PIL with isolated intermittent ankle edema and the absence of overt gastrointestinal symptoms in adulthood. Diagnosis required a high index of suspicion and was facilitated by antegrade double-balloon enteroscopy.

PIL normally presents in childhood, and in a recent review of 84 PIL cases, $53 \%$ of cases occurred before the age of 2 years [2]. Delayed diagnosis can contribute to adult cases and an interval as long as 40 years between the onset of symptoms 


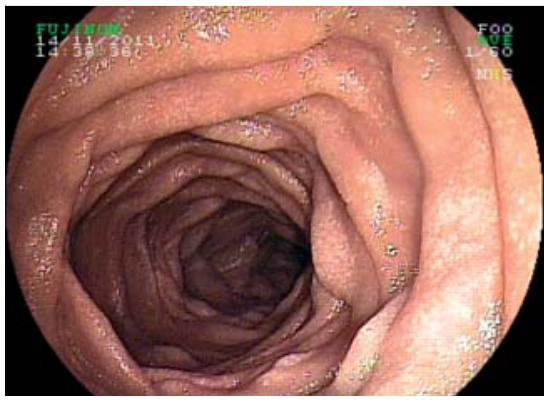

Fig. 1 Edematous jejunal folds at doubleballoon enteroscopy.

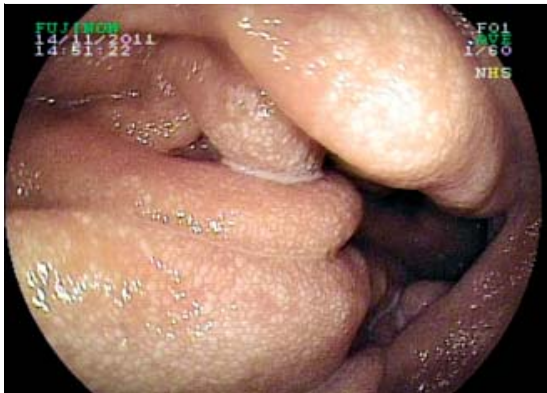

Fig. 2 Free lymph extruding from jejunal villi at double-balloon enteroscopy.

and diagnosis with PIL has been reported [3]. Impaired lymphatic drainage in PIL may be confined to the gastrointestinal tract or form part of a more generalized systemic disorder [4]. Other conditions associated with secondary lymphatic obstruction must be excluded $[1,2]$.

In the unusual setting of adult-onset PIL, clinical features are similar to those found in children [2]. The most common clinical manifestation is peripheral edema secondary to impaired lymphatic drainage and/or hypoalbuminemia [2,5]. Protein-losing enteropathy, steatorrhea, and chylous ascites or pleural effusions may be seen $[2,3,5]$. Lymph leakage into the intestinal lumen leads to hypoalbuminemia, lymphopenia, and hypogammaglobulinemia $[1,2,5]$. Electrolyte disturbance including hypocalcemia and malabsorption of fat-soluble vitamins may occur $[5,6]$. Osteomalacia and osteoporosis as a consequence of malnutrition have been reported [6]. PIL induced immunodeficiency may predispose to opportunistic infection and an initial presentation with cryptococcal meningitis has been reported in a 30 -year-old woman $[4,7]$.

Cross-sectional imaging often demonstrates nonspecific small bowel thickening but is important in localizing abnormalities and excluding a secondary obstructive cause [1,5]. Capsule endoscopy and antegrade double-balloon enteroscopy are increasingly used in the diagnosis of PIL and can identify several abnormalities [1,3, 5, 8-11]. The small bowel endoscopic appearance typically includes edematous mucosal folds, scattered white-tipped villi or spots, white plaques, and the presence of intraluminal chyle-like substance [1,3,5,8-11]. Cystic nodules have also been described [5]. Small-bowel biopsies confirm the diagnosis and demonstrate dilated mucosal and sometimes submucosal lymphatics $[1,5]$.

Treatment is primarily dietary with a low fat, high protein diet supplemented by medium-chain triglycerides $[2,10,12]$. Medium-chain triglycerides are directly absorbed in the portal venous system and so do not exacerbate lacteal engorgement and lymph leakage in the gastrointestinal tract $[2,12]$. Dietary therapy seems less effective in adults and efficacy may be as low as 39\% [2]. The goals of dietary therapy include the resolution of symptoms and signs, and correction of hypoproteinemia and any other

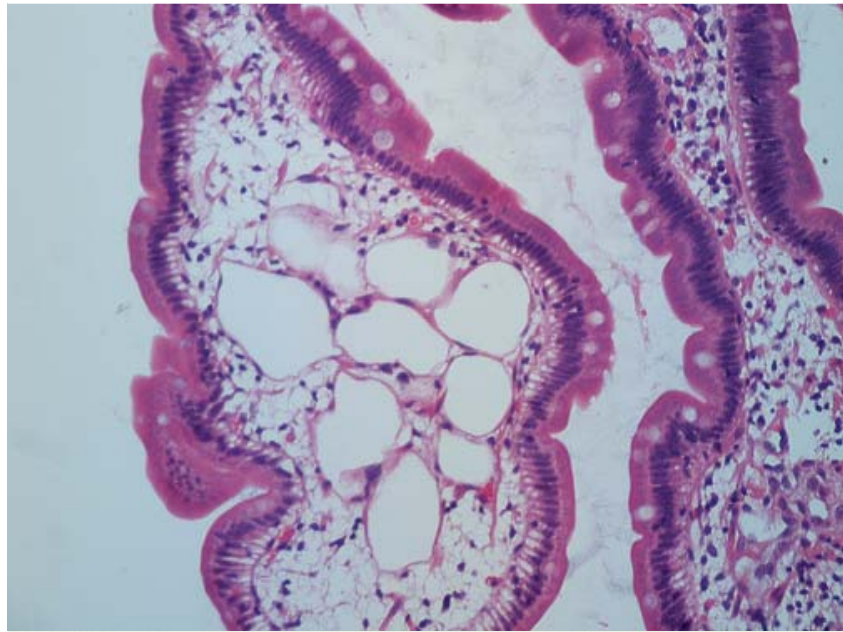

Fig. 3 Biopsy of the jejunal mucosa demonstrating jejunal villous with dilated lymphatics (magnification $\times 20$ ).

biochemical abnormalities. In severe refractory cases, the use of parenteral nutrition can complement enteral nutrition and reduce dietary fat intake $[2,12]$. Surgical resection of localized segmental disease has also been undertaken [2]. The use of octreotide and antiplasmin in cases refractory to dietary therapy has been successful in isolated reports but requires further evaluation $[2,13,14]$. Small intestinal B-cell lymphoma has been reported in a small number of patients with longstanding PIL and while causality cannot be determined through isolated case reports, it has been postulated that this may represent a real phenomenon related to PIL induced immune deficiency $[2,4,15]$.

In summary, PIL is rare and may present insidiously in adults. The diagnosis should be considered in patients with ankle edema and a protein losing enteropathy after exclusion of other more common causes of malabsorption and secondary lymphatic obstruction. Gastroenterologists should also remain vigilant for the development of small-bowel lymphoma in adult patients with PIL.

\section{Competing interests: None}

\section{References}

1 Oh TG, Chung JW, Kim HM et al. Primary intestinal lymphangiectasia diagnosed by capsule endoscopy and double balloon enteroscopy. World J Gastrointest Endosc 2011; 3: 235-240

2 Wen J, Tang $Q W u J$ et al. Primary intestinal lymphangiectasia: Four case reports and a review of the literature. Dig Dis Sci 2010; 55: $3466-3472$

3 Choi EH, Reidel W, Coyle W. Forty years of shortness of breath and lower extremity edema. Gastroenterology 2011; 141: e6 - e7

4 Bouhnik Y, Etienney I, Nemeth J et al. Very late onset small intestinal B cell lymphoma associated with primary intestinal lymphangiectasia and diffuse cutaneous warts. Gut 2000; 47: 296 - 300

5 Salomons HA, Kramer P, Nikulasson S et al. Endoscopic features of longstanding intestinal lymphangiectasia. Gastrointest Endosc 1995; 41: $516-518$

6 Li XP, Shen WB, Long MQ et al. Osteomalacia and osteoporosis associated with primary intestinal lymphangiectasis. Chin Med J (Engl) 2012; 125: $1836-1838$

7 Jabeen SA, Murthy A, Kandadai RM et al. Cryptococcal meningitis as a primary manifestation in a patient with intestinal lymphangiectasia. Ann Indian Acad Neurol 2012; 15: 218-220

8 Ersoy 0 , Akin E, Demirezer A et al. Evaluation of primary intestinal lymphangiectasia by capsule endoscopy. Endoscopy 2013; 45: E61 -E62

9 Fang $Y H$, Zhang $B L, W u J G$ et al. A primary intestinal lymphangiectasia patient diagnosed by capsule endoscopy and confirmed at surgery: A case report. World J Gastroenterol 2007; 13: 2263 -2265 
10 Lai $Y, Y u T$, Qiao $X Y$ et al. Primary intestinal lymphangiectasia diagnosed by double-balloon enteroscopy and treated by medium-chain triglycerides: a case report. J Med Case Rep 2013; 7: 19

11 Asakura H, Miura S, Morishita T et al. Endoscopic and histopathological study on primary and secondary intestinal lymphangiectasia. Dig Dis Sci $1981 ; 26: 312-320$

12 Aoyagi $K$, Iida M, Matsumoto T et al. Enteral nutrition as a primary therapy for intestinal lymphangiectasia: value of elemental diet and polymeric diet compared with total parenteral nutrition. Dig Dis Sci 2005; 50: $1467-1470$
13 Kuroiwa G, Takayama T, Sato Y et al. Primary intestinal lymphangiectasia successfully treated with octreotide. J Gastroenterol 2001; 36: $129-132$

14 MacLean JE, Cohen E, Weinstein M. Primary intestinal and thoracic lymphangiectasia: a response to antiplasmin therapy. Paediatrics 2002; 109: $1177-1180$

15 Patel KV, Goel RM, Wong T. Diffuse large B-cell lymphoma recurrence complicating Primary Intestinal Lymphangiectasia. Clin Gastroenterol Hepatol 2013; 11: e86-e87 